\title{
Spatiotemporal Evolution and Pattern Differences of Environmental Sanitation Facilities in Rural China: Taking the Improvement of Water and Latrines as an Example
}

\author{
Xinjie Deng $\dagger$ \\ Yiwu Industrial and Commercial College, Yiwu Zhejiang 322000, China \\ †Corresponding author: 24250987@qq.com
}

\section{Nat. Env. \& Poll. Tech. \\ Website: www.neptjournal.com \\ Received: 01-02-2021 \\ Revised: 25-04-2021 \\ Accepted: 28-04-2021 \\ Key Words: \\ Rural sanitation facilities Spatiotemporal evolution Improvement of latrines GWR model}

\begin{abstract}
Based on the panel data of water and latrine improvement in rural China from 2003 to 2016, this paper explores the spatiotemporal evolution pattern of rural sanitation facilities and analyzes the spatial heterogeneity of influencing factors of rural sanitation facilities by using the Geographically Weighted Regression (GWR) model. The conclusions are as follows: the gap between the western and the eastern regions of China is gradually narrowing; the spatial differences of rural environmental sanitation facilities in provinces were obvious, showing high-high and low-low agglomeration types. Additionally, years of education per capita, population density, and government investment all have a significant positive impact on the improvement of water and latrines. And the proportion of the minority population has a significant negative impact on the improvement of water. The net income per capita, traffic density, and residential investment per capita are significantly positively correlated with the improvement of water and latrines. But the difference is that the impact on the improvement of water is an obviously east-west band and decreases successively, and the impact on the improvement of latrines shows a dual pattern of polarization between north and south.
\end{abstract}

\section{INTRODUCTION}

The lack of environmental health services in rural areas is an important cause of the health inequality between urban and rural residents, which will have an important impact on the development of rural human capital, and may lead to the further expansion of the income gap between urban and rural areas. Rural environmental sanitation differs due to differences in geographic environment, natural endowments, and socio-economic conditions. Therefore, clarifying the conditions of rural environmental sanitation facilities and the influencing factors of their spatial differentiation has important theoretical and practical value for formulating differentiated policies to promote the equalization of environmental sanitation public services.

The research on rural environmental sanitation facilities has always been a hot topic of continuous research in various countries. The initial research contents included the investigation on the status quo of rural environmental sanitation facilities (Tao 2009), health impact (Miao 2008, Andres et al. 2017, Jalan \& Ravallion 2003, Zhang 2012), willingness to pay for improvement (Abramson et al. 2011, Miao et al. 2012) and equity research (Yang et al. 2013, Phansalkar 2007). Later, studies involving regional differences or spatial distribution of rural environmental sanitation facilities gradually emerged. Some scholars used the method of statistical description to reveal the provincial differences between rural drinking water and sanitary latrines and the correlation between them and socioeconomic development (Wenjie 2019). Subsequently, the geographic information system (GIS) and spatial analysis methods were gradually applied to the study. Scholars either included environmental sanitation facilities in public services (Ma et al. 2011), physical capital (Liu et al. 2017), or human settlement environment indicators (Zhu et al. 2015) to analyze their spatial differentiation pattern and agglomeration trend or analyze the spatially related characteristics and causes of a certain type of environmental sanitation facilities (Wan et al. 2010).

The existing results provide an important theoretical basis and methodological reference for further research. However, the rural environmental sanitation facilities as a whole are rarely studied. Moreover, GIS and spatial analysis methods overemphasize the content of geographic maps, exaggerate the existing problems, and do not fully reflect the real situation such as influencing factors (Lejano 2008). However, the geographically weighted regression (GWR) model can reflect the spatial differences of the influencing factors of the research objects, which takes both influencing 
factors and spatial characteristics into account. In addition, in terms of model construction and data, scholars either used time series or panel data for global regression, often ignoring the existence of spatial nonstationarity, or the GWR model was used to process the spatial cross-section data, ignoring the time dimension. Based on the "individual effect" analysis of the spatial panel data of water and latrine improvement in 30 provinces in China from 2003 to 2016, this paper uses the GWR model to analyze the economic, geographical and social factors affecting the environmental sanitation facilities, and explores the spatiotemporal differentiation characteristics and influencing factors of the environmental sanitation facilities in rural China. This enriches the spatial analysis methods of related research, and in practice provides a basis for policymaking to improve the level of environmental sanitation public services in rural areas.

\section{MATERIALS AND METHODS}

This paper conducts an empirical study on the panel data of 420 observational values from 2003 to 2016 in 30 provinces of China (except Tibet, and not including Hong Kong, Macao, and Taiwan). Data was obtained from China Statistical Yearbook, China Rural Statistical Yearbook, China Health Statistical Yearbook, China Environmental Statistical Yearbook, China Population, and Employment Statistical Yearbook, and China Transport Statistical Yearbook. Missing data was supplemented by the trend extrapolation method. The name, unit, and statistical description of each variable are shown in Table 1. The abbreviation for the variable is in parentheses.

Spatial autocorrelation analysis: When there is a potential interdependence between the observed data of some variables in the same distribution region, the spatial autocorrelation method can be used for spatial analysis. Based on the spatial autocorrelation model, this paper analyzes the spatial agglomeration characteristics of water and latrine improvement in 30 provinces of China. Spatial autocorrelation analysis includes two aspects, one is global spatial autocorrelation analysis. It is used to test whether there is a correlation among neighboring provinces. Moran's I index can be used to analyze the study area, and its formula is,

$$
I=\frac{n}{\sum_{i} \sum_{j} W_{i j}} \cdot \frac{\sum_{i} \sum_{j} W_{i j}\left(x_{i}-\bar{x}\right)\left(x_{j}-\bar{x}\right)}{\sum_{i}\left(x_{i}-\bar{x}\right)^{2}}
$$

Here, $n$ is the number of provinces, and $W_{i j}$ is the spatial adjacent weight matrix of $i$ and $j$ of each province in China. When the two are adjacent, $W_{i j}$ is 1 ; otherwise, it is 0 . And $x_{i}$ is the mean of the observed value of $i$ province. At a given significance level, Moran's I is greater than 0, indicating a positive correlation. Otherwise, it indicates a negative correlation. The higher the value is, the higher the correlation degree is, and vice versa. Moran's I equal zero means the distribution is random.

The second is the local spatial autocorrelation analysis. This analysis method can be used to test the degree of spatial correlation and spatial difference of the study area. In this paper, the Moran scatter plot is used to measure the spatial local correlation. Its formula (Anselin 1995) is as follows,

$$
I_{i}=\frac{\left(x_{i}-\bar{x}\right) \sum_{j} W_{i j}\left(x_{i}-\bar{x}\right)}{\sum_{i}\left(x_{i}-\bar{x}\right)^{2} / n}
$$

Geographically Weighted Regression (GWR) model: GWR model is an extension of the ordinary linear regression model and a new method used to study the complexity, autocorrelation, and variability of spatial data. Its purpose is to explore the influence of independent variables on dependent variables with spatial changes. Fotheringham et al. (1998) embedded the spatial position of the data into the regression parameters and performed point-by-point parameter estima-

\begin{tabular}{|c|c|c|c|c|c|}
\hline Variables & Unit & Mean & Std. dev. & Min & Max \\
\hline Cumulative benefit rate of water improvement (Benefit_water) & $\%$ & 93.92 & 7.605 & 55.41 & 100 \\
\hline Cumulative benefit rate of latrine improvement (Benefit_latrine) & $\%$ & 64.30 & 18.30 & 21.50 & 99.80 \\
\hline Net income per capita (NIPC) & Yuan per person & 6949 & 4339 & 1565 & 26000 \\
\hline Years of education per capita (YEPC) & Year & 7.380 & 0.688 & 5.139 & 9.304 \\
\hline Population density (PD) & People. $\mathrm{km}^{-2}$ & 207.8 & 149.6 & 3.973 & 734.6 \\
\hline Traffic density (TD) & $\mathrm{km} \cdot \mathrm{km}^{-2}$ & 0.682 & 0.474 & 0.0310 & 2.184 \\
\hline Cumulative investment in water improvement per capita (Invest_water) & Yuan per person & 785.0 & 1123 & 0 & 8755 \\
\hline Cumulative investment in latrine improvement per capita (Invest_latrine) & Yuan per person & 84.47 & 115.9 & 0 & 590.6 \\
\hline Cumulative residential investment per capita (CRIPC) & Yuan per person & 3471 & 3382 & 73.38 & 21000 \\
\hline Annual average temperature (AAT) & Centigrade & 14.52 & 5.072 & 4.300 & 25.40 \\
\hline Annual precipitation in major cities (APMC) & $\mathrm{Mm}$ & 909.2 & 528.1 & 0 & 2940 \\
\hline The proportion of minority population (PMP) & $\%$ & 12.36 & 15.85 & 0.260 & 59.52 \\
\hline
\end{tabular}

Table 1: Variable statistics and variable description. 
tion using the locally weighted least square method. It should be pointed out that the weight here is the distance function between the geospatial position of the regression point and the geospatial position of other observation points. The regression coefficient changes with the change of the spatial position, and the formula are as follows (Cui et al. 2012):

$$
y_{i t}=\propto_{i}+\beta_{0}\left(u_{i}, v_{i}\right)+\sum_{k=1}^{n} \beta_{k}\left(u_{i}, v_{i}\right) X_{i k t}+\varepsilon_{i t}
$$

In the formula, $y_{i t}$ is the cumulative benefit rate of water or latrine improvement in each province every year; $a_{i}$ represents the individual fixed effect vector. The subscript $i$ of all variables represents the individual and $t$ represents the time. Symbol $\left(u_{i}, v_{i}\right)$ is the coordinate of $i$ province, and $\mathrm{b}_{k}\left(u_{i}, v_{i}\right)$ is the regression coefficient of the $k$ variable of $i$ province. $X_{i k t}$ is the explanatory variable matrix, and $\mathrm{e}_{i t}$ is the error term, obeying the normal distribution with constant variance. Since spatial panel data is used in this paper, each variable has two attributes of both time dimension and space dimension.

With regard to the selection of explanatory variable $X_{i k t}$ in the GWR model, existing literature has emphasized the socio-economic and cultural factors at the family level (Cheng \& Wang 2006), while this paper mainly discusses the geographical environment and socio-economic factors at the regional level. Some scholars have proposed that income, the proportion of ethnic minorities, education level, and average temperature in winter will affect the local environmental sanitation level (Miao \& Chen 2016), and government investment in environmental sanitation facilities also plays a decisive role (Peng \& Lu 2010). In addition, since this paper focuses on the spatial heterogeneity of rural environmental sanitation facilities, the spatial network characteristics of population distribution and roads are the most obvious. Therefore, they are included in the explanatory variable of the GWR model in this paper. Taking into account China's special national conditions, the distribution characteristics of rural sanitation facilities, and the availability of data, this article finally selects 9 indicators including net income per capita, years of education per capita, population density, traffic density, government investment in water improvement (or latrine improvement) per capita, cumulative investment per capita in residential buildings, annual average temperature, annual precipitation in major cities, and proportion of ethnic minority populations as explanatory variables.

\section{RESULTS AND DISCUSSION}

Spatiotemporal changes of rural water and latrines in China: According to the natural discontinuity method, the coverage rate of water improvement and latrine improvement is divided into four levels: low, lower, higher, and high. For comparison of water improvement, two time sections in 2005 and 2014 were taken. While for comparison of latrine improvement, two time sections in 2005 and 2016 were taken, as shown in Fig. 1 and Fig. 2. In terms of water improvement, Beijing, Tianjin, and Shanghai are at a high level all the time. Hebei, Shandong, Jiangsu, and Guangxi were upgraded from a higher level to a high level. Heilongjiang, Jilin, Hubei, and Jiangxi rose from a higher level to a high level. The western provinces of Xinjiang, Gansu, and Inner Mongolia saw a rapid increase in the benefit rate of water improvement. From the perspective of latrine improvement, the provinces with a high level of benefit of latrine improvement have moved from the central and eastern regions to the eastern coastal areas.

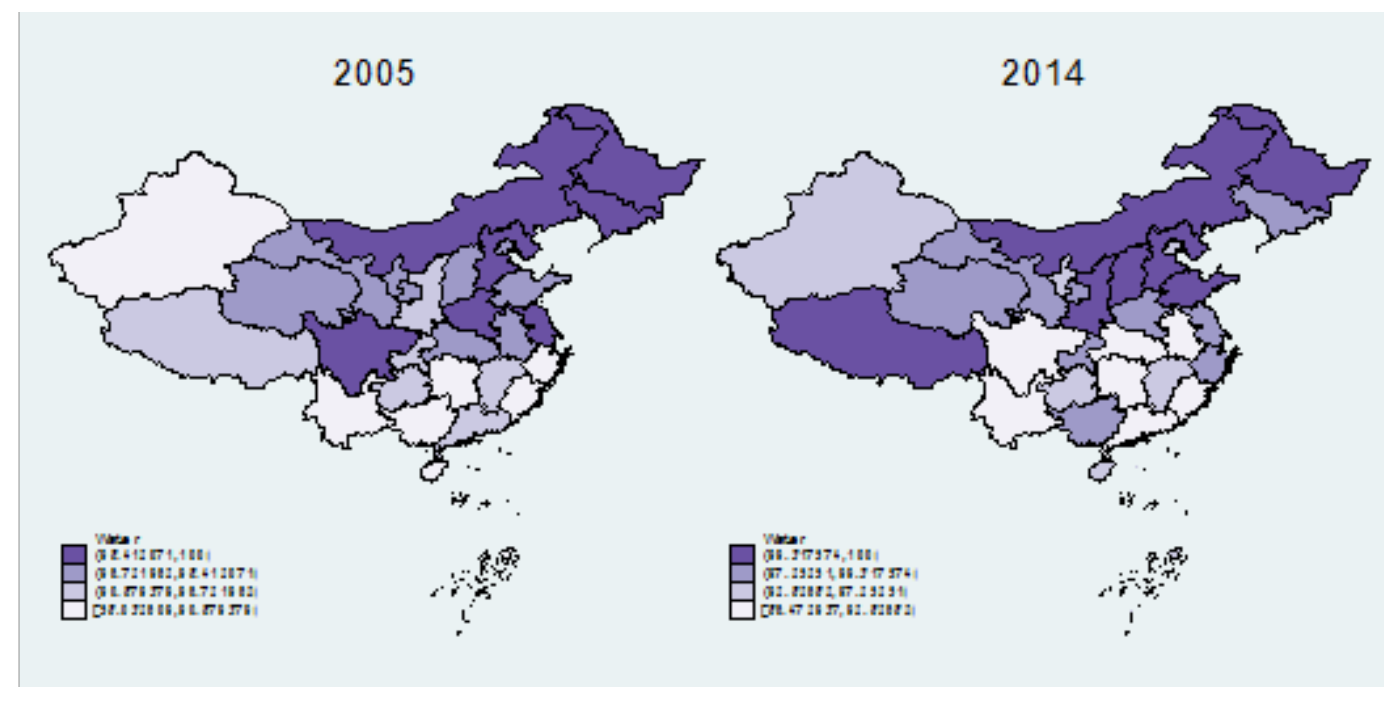

Fig. 1: Spatial evolution of water improvement in rural China in 2005 and 2014. 


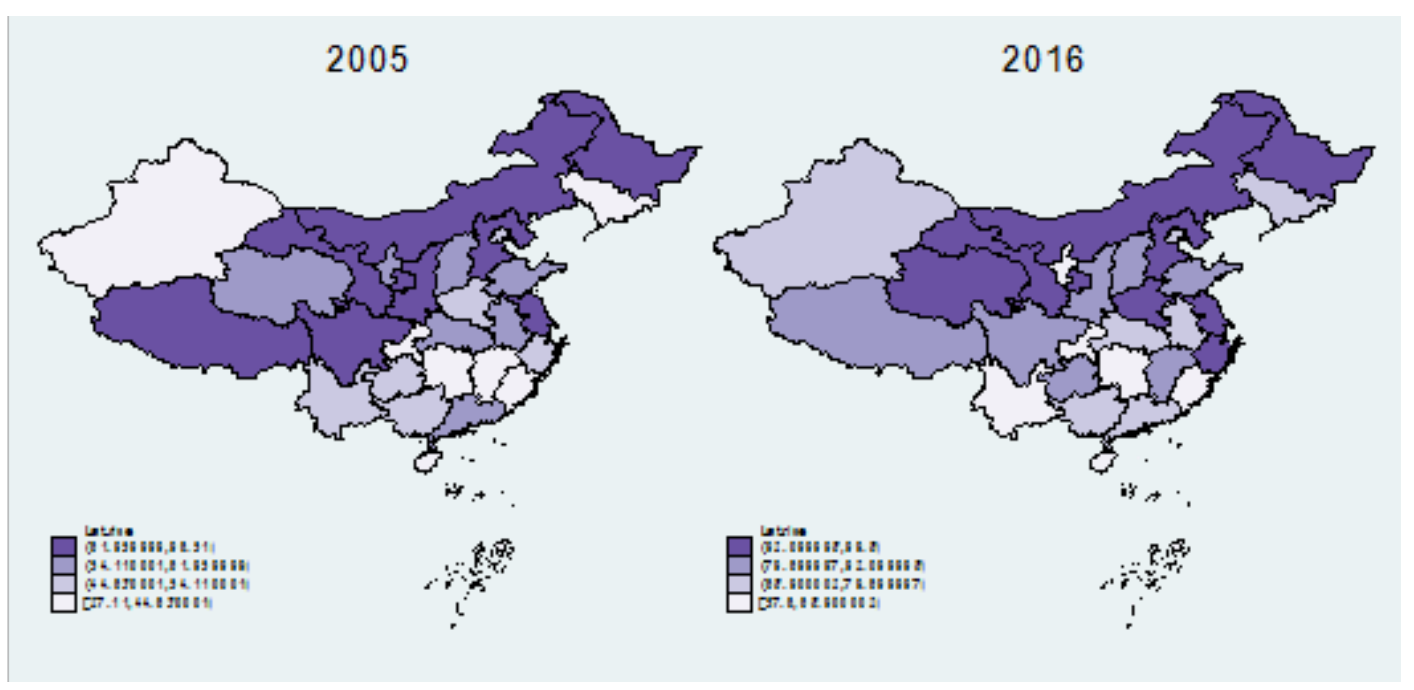

Fig. 2: Spatial evolution of latrine improvement in rural China in 2005 and 2016.

The specific performance is: Hubei, Jiangxi, and Guangxi have been reduced from high-level to higher-level areas; Three provinces of Guangdong, Fujian, and Jiangsu have been upgraded from high-level to higher-level areas; The three municipalities, Shandong and Zhejiang have always been at a high level. Generally speaking, the provinces with higher coverage of rural sanitation facilities in China are located in the northeast and eastern coastal areas, and the rural sanitation facilities in the central provinces are divided. The provinces in the western region are currently at the same level as the central provinces with rapid progress, and the gap with the eastern provinces is gradually narrowing.

Spatial autocorrelation analysis: The global autocorrelation index Moran's I of water improvement in 2014 and latrine improvement in 2016 was calculated using Stata13 software, global autocorrelation model, and formula (1).
The values are 0.25 and 0.35 , and the significance levels are 0.01 and 0.001 , respectively. Z (I) is also greater than the critical value of 2.58 . This indicates that there is a spatial correlation between rural environmental sanitation facilities in China. Global Moran's I can examine the overall relevance of space, but it will cover up its local spatial heterogeneity (Whittington et al. 1993). While local Moran's I scatter plot can reveal the differences in regional economic development. Therefore, local Moran's I scatter plots of water improvement in 2005 and 2014 and latrines improvement in 2005 and 2016 (Fig. 3 and Fig. 4) were extracted in this paper to explore the spatial relevance and development imbalance of local environmental sanitation in rural China.

According to Moran's I scatter plot, the spatial correlation degree of environmental sanitation facilities between a province and its neighboring provinces can be divided
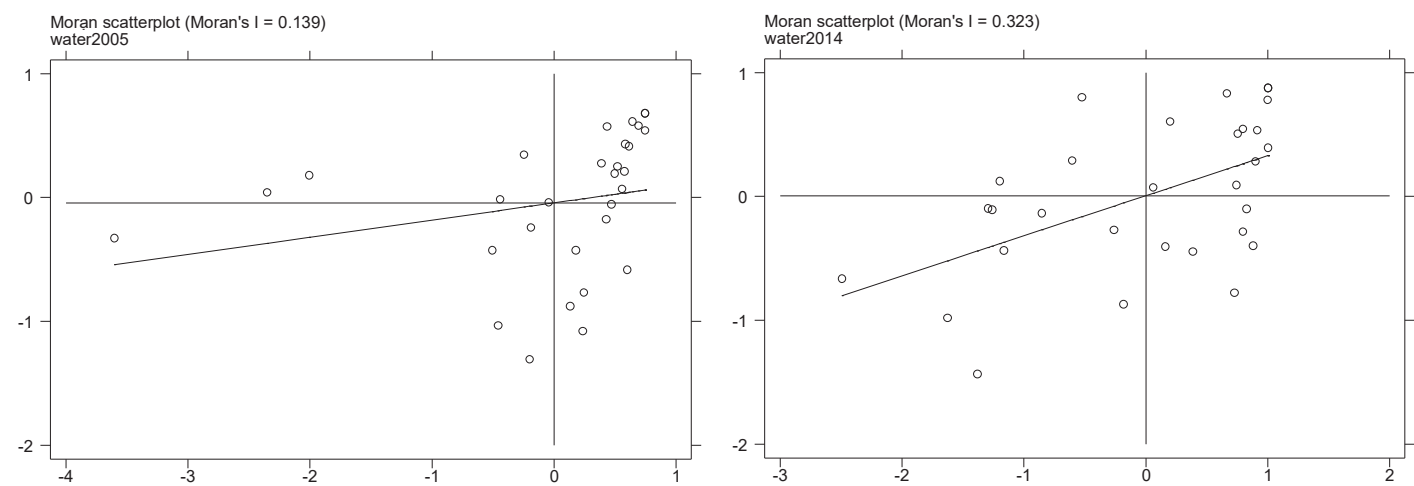

Fig. 3: Local Moran's I scatter plot of rural water improvement in China in 2005 and 2014. 

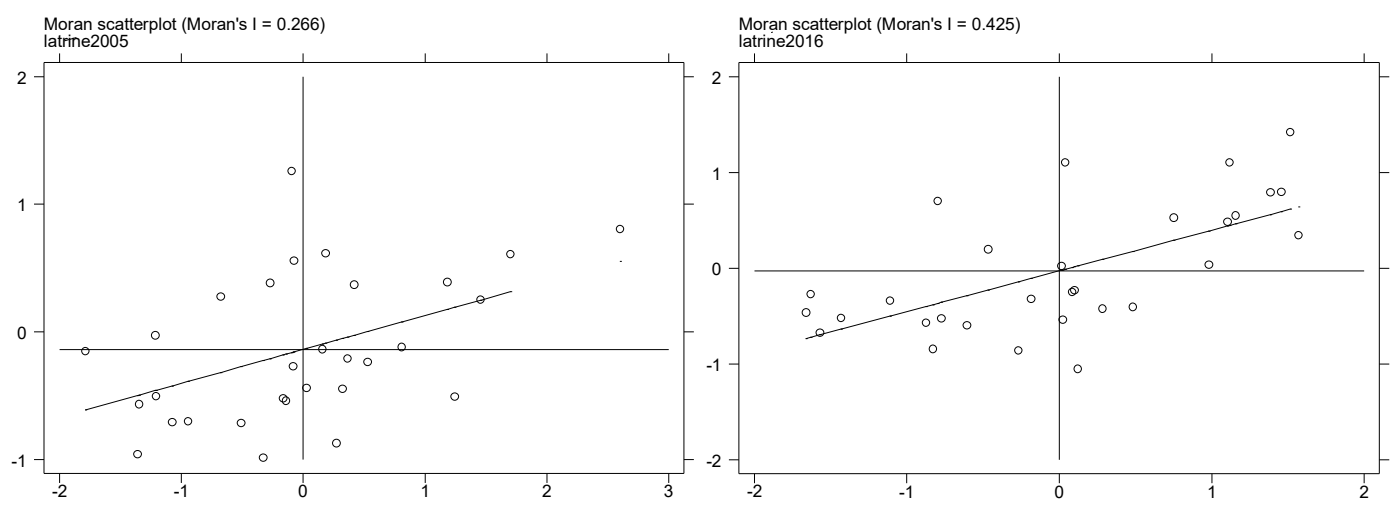

Fig. 4: Local Moran's I scatter plot of latrine improvement in rural China in 2005 and 2016.

into four types. In the first quadrant, the spatial difference is small, and the coverage rate of environmental sanitation facilities is high, which belongs to the "high-high (H-H)" type. In the third quadrant, the spatial difference is small, and the coverage rate of environmental sanitation facilities is low, which belongs to the "low-low (L-L)" type. In the second quadrant, there is a large spatial difference between a province and its neighboring provinces. The province itself has a high coverage rate of environmental sanitation facilities, while the neighboring provinces have a low coverage rate, which belongs to the "high-low (H-L)" type. In the fourth quadrant, there is also a large spatial difference between a province and its neighboring provinces, and the province itself has a low coverage rate, while the neighboring provinces have a high coverage rate, which belongs to the "low-high (L-H)" type. As can be seen from Fig. 3, the number of regions distributed in the first quadrant is highly dense in both time sections. That is, the $\mathrm{H}-\mathrm{H}$ type occupies the superiority in quantity, but the intensity is reduced to a certain extent. And the distribution location is no longer so concentrated. The number of regions in the third quadrant begins to increase. This indicates that the gap between the eastern and western regions of rural water reform has been alleviated, but it still cannot change the dual pattern. As can be seen from Fig. 4, the number of areas distributed in the first and third quadrants shows an increasing trend. In conclusion, rural environmental sanitation facilities in China show agglomeration characteristics, which is consistent with the analysis results of the spatiotemporal evolution.

\section{Analysis of influencing factors of the spatial distribution of rural environmental sanitation facilities: Through} the above autocorrelation analysis, rural environmental sanitation facilities in China have spatial autocorrelation and significant regional differences. To further explore its formation mechanism, this paper uses the GWR model to analyze its influencing factors by using provincial spatial panel data of rural environmental sanitation facilities in China from 2003 to 2016.

Considering the reference frame, mixed regression was first carried out, and the regression results are shown in the OLS model in Table 2. Due to the different conditions of each province, there may be missing variables that do not change over time, so the fixed-effect (FE) model is considered. The output also contains an $F$-test with the null assumption that the individual effect $u_{i}$ for all provinces is 0 . Since the $p$-value of the regression results of fixed effect of water and latrine improvement is $0.000 \mathrm{in} F$-test, the null hypothesis is strongly rejected. That is, the individual effect of provinces exists, and mixed regression should not be used. Although the above results have basically confirmed the existence of individual effects in provinces, individual effects may still exist in the form of random effects (RE). After the regression results of the random effects of water and latrine improvement and the LM test to test the individual effects, the LM test strongly rejects the null hypothesis that "there is no individual random effect". So there are also individual random effects of provinces. Hausmann's test is used to determine whether to use fixed-effects or random-effects models. But Hausmann's test results cannot strongly reject the null hypothesis " $\mathrm{H} 0: u_{i}$ is not related to $x_{i t}$ or $z_{i}$ ". Therefore, the random effects of provinces should be considered. Because the "GWR" come mand in Stata13 software does not have " $r e$ " or " $f e$ " options, spatial panel data cannot be processed. In this paper, each variable was transformed by "random effect", and then the GWR model was carried out. See Table 2 for the regression results of water and latrine improvement.

The geographically weighted regression analysis of water improvement: See Fig. 5 and Fig. 6 for the output of the GWR model. Among the regression coefficients of various factors affecting water improvement, the absolute value of the coefficient of years of education per capita is the largest, which is significant at the level of $5 \%$. Years of education 
Table 2: Regression results of influencing factors of water and latrine improvement in rural China.

\begin{tabular}{|c|c|c|c|c|c|c|c|c|}
\hline & \multicolumn{4}{|l|}{ Water } & \multicolumn{4}{|l|}{ Latrine } \\
\hline & OLS & $\mathrm{FE}$ & $\mathrm{RE}$ & GWR & OLS & $\mathrm{FE}$ & $\mathrm{RE}$ & GWR \\
\hline \multirow[t]{2}{*}{ NIPC } & $0.000^{* *}$ & -0.000 & -0.000 & -0.000 & $0.002^{* * * *}$ & $0.001^{* * * *}$ & $0.001^{* * * *}$ & $0.001^{* * * *}$ \\
\hline & $(0.000)$ & $(0.000)$ & $(0.000)$ & $(0.000)$ & $(0.000)$ & $(0.000)$ & $(0.000)$ & $(0.001)$ \\
\hline \multirow[t]{2}{*}{ YEPC } & 0.615 & $2.442^{* *}$ & $2.009^{* *}$ & $2.192^{* *}$ & 0.436 & $2.740^{* *}$ & $2.456^{* *}$ & $2.499^{* *}$ \\
\hline & $(0.576)$ & $(0.982)$ & $(0.818)$ & $(0.859)$ & $(0.959)$ & (1.369) & $(1.240)$ & (1.249) \\
\hline \multirow[t]{2}{*}{ PD } & $0.010^{* * * *}$ & 0.012 & $0.011^{* *}$ & $0.011^{* *}$ & $0.030^{* * *}$ & $0.027^{* *}$ & $0.027^{* * *}$ & $0.026^{* * *}$ \\
\hline & $(0.004)$ & $(0.007)$ & $(0.005)$ & $(0.005)$ & $(0.006)$ & $(0.010)$ & $(0.008)$ & (0.008) \\
\hline \multirow[t]{2}{*}{$\mathrm{TD}$} & -1.948 & -1.023 & -1.369 & -1.257 & -0.378 & $6.178^{* *}$ & $5.282^{* *}$ & $5.405^{* *}$ \\
\hline & (1.456) & $(1.864)$ & $(1.620)$ & $(1.667)$ & $(2.482)$ & $(2.442)$ & $(2.272)$ & $(2.277)$ \\
\hline \multirow[t]{2}{*}{ Invest_water } & 0.000 & $0.002^{* * * *}$ & $0.001^{* * *}$ & $0.001^{\text {**** }}$ & $0.025^{\text {**** }}$ & $0.023^{* * * *}$ & $0.022^{* * * *}$ & $0.022^{* * * *}$ \\
\hline & $(0.000)$ & $(0.000)$ & $(0.000)$ & $(0.001)$ & $(0.006)$ & $(0.009)$ & $(0.008)$ & $(0.008)$ \\
\hline \multirow[t]{2}{*}{ Invest_latrine } & 0.000 & 0.000 & 0.000 & 0.001 & $0.001^{* *}$ & $0.001^{* * *}$ & $0.001^{* * * *}$ & $0.001^{* * *}$ \\
\hline & $(0.000)$ & $(0.000)$ & $(0.000)$ & $(0.000)$ & $(0.000)$ & $(0.000)$ & $(0.000)$ & $(0.001)$ \\
\hline \multirow[t]{2}{*}{ AAT } & $-0.257^{* * *}$ & 0.014 & -0.139 & -0.100 & $-0.574^{* * *}$ & 0.025 & -0.035 & -0.026 \\
\hline & $(0.097)$ & $(0.190)$ & $(0.137)$ & $(0.149)$ & $(0.166)$ & $(0.259)$ & $(0.214)$ & $(0.218)$ \\
\hline \multirow[t]{2}{*}{ APMC } & $0.002^{*}$ & 0.001 & 0.001 & 0.001 & $0.009^{* * * *}$ & 0.001 & 0.002 & 0.002 \\
\hline & $(0.001)$ & $(0.001)$ & $(0.001)$ & $(0.001)$ & $(0.002)$ & $(0.001)$ & $(0.001)$ & $(0.001)$ \\
\hline \multirow[t]{2}{*}{ PMP } & $-0.226^{* * * *}$ & 0.000 & $-0.223^{* * *}$ & $-0.220^{* * * *}$ & $-0.093^{* *}$ & 0.000 & -0.053 & -0.051 \\
\hline & $(0.025)$ & $(0.000)$ & $(0.056)$ & $(0.071)$ & $(0.040)$ & $(0.000)$ & $(0.108)$ & (0.116) \\
\hline \multirow[t]{2}{*}{ _cons } & $90.151^{* * * *}$ & $72.205^{* * *}$ & $80.299^{* * * *}$ & $17.758^{* * *}$ & $38.280^{* * *}$ & $19.827^{* *}$ & $22.777^{* *}$ & $4.293^{* * *}$ \\
\hline & $(4.186)$ & (7.223) & $(6.226)$ & $(5.034)$ & $(7.080)$ & (10.065) & $(9.586)$ & (1.855) \\
\hline $\mathrm{N}$ & 420 & 420 & 420 & 420 & 420 & 420 & 420 & 420 \\
\hline $\mathrm{F}$ & 35.139 & 9.810 & 9.69 & 10.387 & 117.999 & 114.687 & 7.201 & 7.834 \\
\hline Quasi-R $^{2}$ & 0.423 & 0.170 & 0.168 & 0.180 & 0.715 & 0.678 & 0.705 & 0.767 \\
\hline
\end{tabular}

Note: The parentheses are the standard deviations of the coefficient. $* * *, * *$ and $*$ indicate that the variable is significant at the level of $1 \%, 5 \%$ and $10 \%$, respectively.

per capita has the greatest impact on water improvement in Xinjiang, Inner Mongolia, Shaanxi, Sichuan, Chongqing, and Guizhou, followed by Shanxi, Anhui, Jiangxi, Henan, Hubei, and Hunan in the central region, and less in Shandong, Jiangsu, Shanghai, Zhejiang, and Fujian in the eastern region. Generally speaking, the impact of education level on rural water improvement in China shows an obvious trend of decreasing in turn. It can be seen that improving the education level of rural residents in underdeveloped areas, especially in the western region, has a significant role in promoting the improvement of safe drinking water facilities. The prof portion of the ethnic minority population is the second most important factor affecting rural water improvement. The larger the value, the lower the coverage rate of rural water improvement. The provinces with the larger absolute value of the impact coefficient mainly include provinces where ethnic minorities live in concentrated communities such as Xinjiang, Guizhou, Hunan, Sichuan, Inner Mongolia, and Qinghai. There is no obvious correlation between rural water improvement and the size of the minority population in the provinces of Northeast and South China.

Population density is the third most important factor affecting rural water improvement. The provinces with a large impact coefficient mainly include the densely populated five provinces of Shanxi, Henan, Hubei, Chongqing, and Sichuan, as well as the sparsely populated western province Xinjiang. The possible reason is that the water improvement project is difficult to construct in places with low population density, and it is easy to develop in places with high population density because of the economies of scale. The benefit rate of rural water improvement is significantly positively correlated with the government investment per capita in water improvement at $1 \%$. Fig. 5 shows the regression coefficient of the government's cumulative water improvement investment per capita, which is the largest in Xinjiang, Sichuan, Chongqing, Guizhou, Guangxi, and Hubei in the west. In other central provinces, eastern and northeastern provinces, 


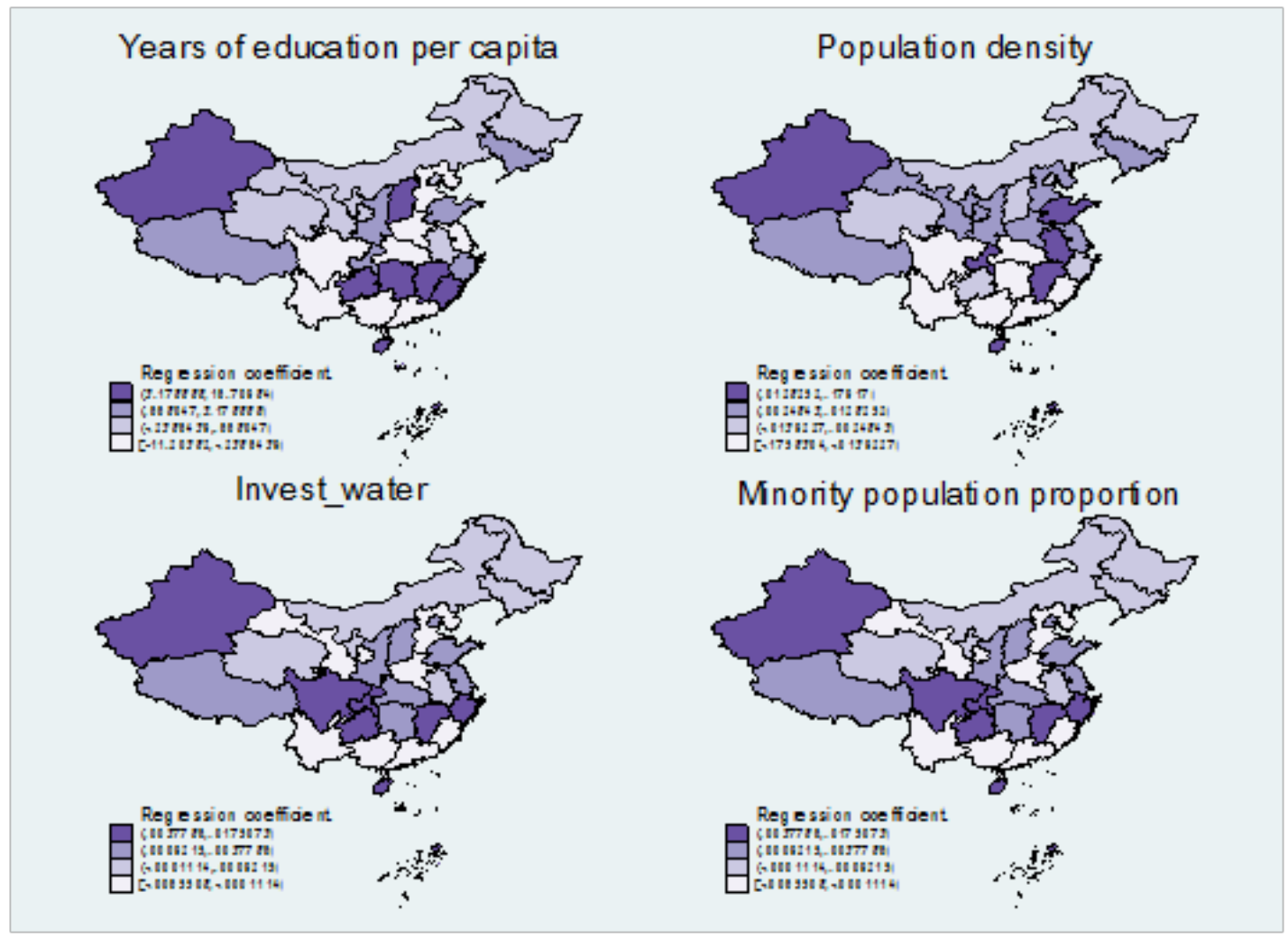

Fig. 5: Distribution of regression coefficients of GWR model for rural water improvement in China.

the regression coefficient is small. It shows that the water improvement cannot be separated from the promotion of the government and the support of government investment funds. In the western provinces, which are relatively undeveloped areas, the improvement of water in rural areas is more dependent on government financial support.

The geographically weighted regression analysis of latrine improvement: The impact of net income per capita on rural latrine improvement is significant at $1 \%$, and an increase in net income per capita of 1,000 Yuan can increase the coverage of rural latrine improvement by $1 \%$, showing a large impact. The provinces with larger regression coeffie cients of net income per capita include Inner Mongolia, Jilin, Shanxi, Anhui, Fujian, Guangxi, and Hainan, involving the four major regions of China. It shows that the impact of net income per capita on latrine improvement is not differentiated by region. Although the years of education per capita has a significant impact on rural latrine improvement at the level of $5 \%$, its impact coefficient is relatively large. High-value areas are mainly distributed in the six provinces of Hubei, Anhui, Jiangsu, Jiangxi, Fujian, and Zhejiang in the middle and lower reaches of the Yangtze River, and Xinjiang in the western region. The low-value areas are distributed in Qinghai, Inner Mongolia, Shanxi, Hebei, and Shandong in the north. It shows that years of education per capita has a polarized dual pattern of impact on rural latrine improvement in the northern and southern provinces. Population density also has a positive impact on the latrine improvement in rural areas, and the highest value areas are mainly found in the northern provinces of Xinjiang, Inner Mongolia, Shaanxi, Heilongjiang, and Liaoning. The difference in latrine habits between northern and southern areas, coupled with the lack of people, makes the incentive to improve latrines even less strong.

The influence of traffic density on rural latrine improvement is significant at the level of $5 \%$, and its regression coefficient is the largest among the six influencing factors. However, there are no obvious regional distribution characteristics between high- and low-value areas of the influence coefficient. It shows that the influence of traffic density is regional, and the improvement of traffic conditions also contributes to the improvement of latrines in rural areas. The average coefficient of the impact of cumulative government investment per capita on latrine improvement in rural areas is 0.022 , indicating that every 100 Yuan increase in cumulative government investment per capita can increase the coverage rate of latrine improvement by $2.2 \%$, which is far greater than the impact of net income per capita. The high-value areas 


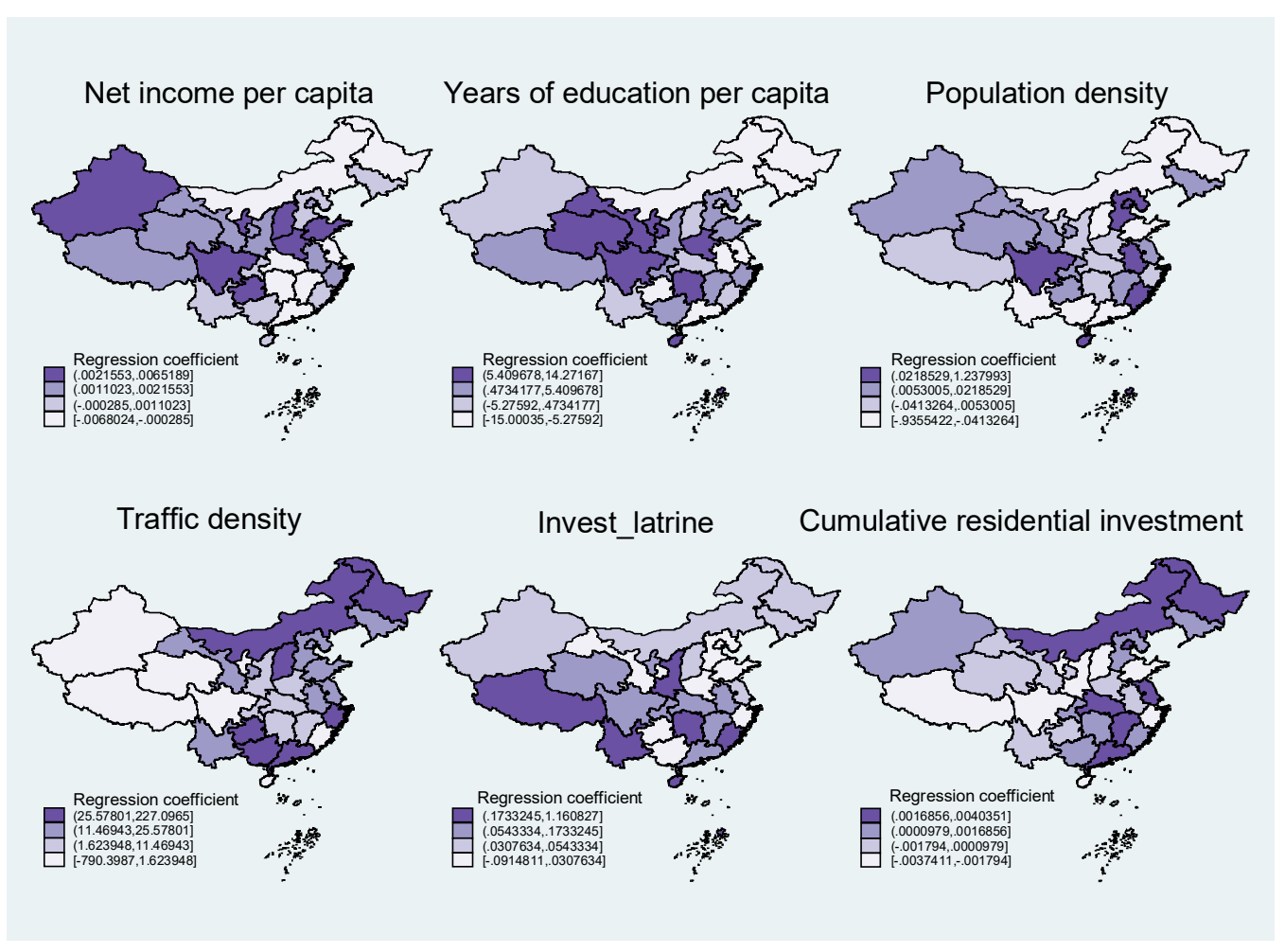

Fig. 6: Regression coefficient distribution of GWR model for latrine improvement in rural China.

are mainly concentrated in the southwest of China, including Yunnan, Guizhou, Hunan, Sichuan, Chongqing, Ningxia, and Shaanxi. The low-value areas are mainly concentrated in the northeast and eastern coastal provinces. The impact of cumulative government investment per capita on rural latrine improvement decreases from west to east in a banding manner. The distribution of the regression coefficient on the map of the impact of the cumulative residential investment per capita is similar to that of the cumulative government investment. The difference is that the overall regression coefficient is far less than that of cumulative government investment per capita in latrine improvement.

\section{CONCLUSIONS}

This article takes China's rural water and latrine improvements as an example, uses spatial autocorrelation and local Moran's I scatter plots to analyze the spatiotemporal evolution characteristics of rural sanitation facilities in China from 2003 to 2016, and uses the GWR model further to analyze the driving factors of the difference in coverage of environmental sanitation facilities from a spatial perspective. The spatial autocorrelation analysis of rural environmental sanitation facilities in China shows that there is a certain spatial correlation and dependence among rural environ- mental sanitation facilities in different provinces, which has obvious spatial agglomeration characteristics, namely high-high agglomeration, and low-low agglomeration. There is a decreasing trend in the low-low type and high-high type of water improvement, while there is an increasing trend in the low-low type and high-high type of latrine improvement.

The analysis results of the GWR model show that among the explanatory variables, there is a significant positive correlation between the number of years of education per capita and the improvement of water and latrines. The difference is that the impact of education level on rural water improvement decreases in an obvious band from west to east, while the impact on rural latrine improvement shows a dual pattern of polarization between the northern and southern provinces. The population density is significantly positively correlated with the improvement of water or latrines. The difference is that it mainly has a significant impact on the improvement of water in northern provinces such as the Northwest and Northeast, and has no obvious impact on the six central provinces. The cumulative government investment in improving water and latrines is the third major factor affecting rural water and latrine improvements in China. The western provinces are more dependent on government investment, while the eastern provinces are less dependent. 
In addition, the proportion of the ethnic minority population is significantly negatively correlated with the improvement of water. The provinces with greater influence are also mainly located in areas where ethnic minorities live. Traffic density and net income per capita have a significant impact on the improvement of latrines.

\section{ACKNOWLEDGMENTS}

We acknowledge the financial support of the Education Department of Zhejiang Province of China (No. FG2020203).

\section{REFERENCES}

Abramson, A., Becker, N., Garb, Y., and Lazarovitch, N. 2011. Willingness to pay, borrow and work for rural water service improvements in developing countries. Water Resour. Res., 47(11): 553-561.

Andres, L., Briceño, B., Chase, C. and Echenique, J.A. 2017. Sanitation and externalities: evidence from early childhood health in rural India. J. Water Sanit. Hyg. Dev., 7(2): 272-289.

Anselin, L. 1995. Local indicators of spatial association: LISA. Geogr. Anal., 27(2): 93-115.

Cheng, P. and Wang, P. 2006. Influences of modes of production and life upon environment and sanitation in ethnic rural areas: taking Chengbu County in Hunan Province, Longsheng County in Guangxi Autonomous Region, Liping in Guizhou Province as the example. J. Cent. South Univ. for National. - Human. Social Sci. Edn., 26(5): 56-59.

Cui, C., Jiang, S. and Zhang, Z. 2012. The analysis of spatial variability of influencing factors to county economy in Hebei based on BGWR. Econ. Geogr., 32(2): 39-45.

Fotheringham, A.S., Charlton, M.E. and Brunsdon, C. 1998. Geographically weighted regression: a natural evolution of the expansion method for spatial data analysis. Environ.Plan A - Econ. Space, 30(11): 19051927.

Jalan, J. and Ravallion, M. 2003. Does piped water reduce diarrhea for children in rural India? J. Econom., 112(1): 153-173.

Lejano, R.P. 2008. Technology and institutions: a critical appraisal of GIS in the planning domain. Science Technology \& Human Values, 33(5): 653-678.
Liu, C., Liu, Y. and Wang, C. 2017. Spatial characteristics of livelihood assets of poor farmers and its influential factors in loess hilly region: a case study of Yuzhong County, Gansu Province. Econ. Geogr., 37(12): 153-162.

Ma, H., Han, Z. and Jiang, H. 2011. The characteristics and spatial differences of basic public services of cities at the prefecture-level and above in China. Econ. Geogr., 31(2): 212-217.

Miao, Y. 2008. Access to health resources and the health problems of farmers: An empirical analysis from rural China. Chin. Popul. Sci., 3: 47-55.

Miao, Y. and Chen, W. 2016. Rural water and environmental sanitation: achievements and challenges. Social Science Literature Press, Beijing, China, pp. 44.

Miao, Y., Yang, Z. and Zhou, H. 2012. Study on willingness to pay for environmental hygiene improvement of rural residents and its influencing factors: taking latrine improvement as an example. Manage. World, 9: 89-99.

Peng, Y. and Lu, Y. 2010. Analysis of the spatio-temporal evolution of the economic development difference in the Chengyu Economic Zone. Econ. Geogr., 30(06): 912-917.

Phansalkar, S. J. 2007. Water, equity and development. Iwmi Res. Rep., 3(3): 1-25.

Tao, Y. 2009. Investigation of drinking water and environmental sanitation in rural China. J. Environ. Health, 26(1): 1-2.

Wan, K., Yu, S. and Hu, Q. 2010. A spatial econometric analysis of rural drinking water safety in Zhejiang. Rural Water Conserv. Hydro. China, 4: 136-139.

Wenjie, Y. 2019. Macroscopic factor decomposition of non-point source pollution of chemical fertilizer: scale, structure, and constraint. Nature Environ. Pollut. Technol., 18(1): 323-327.

Whittington, D. 1993. Household demand for improved sanitation services in Kumasi, Ghana: a contingent valuation study. Water Resour. Res., 29(6): 1539-1560.

Yang, H., Bain, R., Bartram, J., Gundry, S., Pedley, S. and Wright, J. 2013. Water safety and inequality in access to drinking water between rich and poor households. Environ. Sci. Technol., 47(3): 1222.

Zhang, J. 2012. The impact of water quality on health: evidence from the drinking water infrastructure program in rural China. J. Health Econ., 31(1): 122-134.

Zhu, B., Zhang, X. and Yin, X. 2015. Evaluation of rural human settlements quality and its spatial pattern in Jiangsu Province. Econ. Geogr., 35(3): 138-144. 